\title{
A Single Retrograde Revascularization onto the Superior Mesenteric Artery Using an Artificial Graft for Abdominal Angina: A Case Report
}

\author{
Ai Tochikubo, MD, ${ }^{1}$ Shinji Abe, MD, ${ }^{1}$ Tomoji Yamakawa, MD, ${ }^{1}$ Makoto Yoshida, MD, ${ }^{2}$ \\ Yasushige Shingu, MD, PhD, ${ }^{3}$ and Yoshiro Matsui, MD, $\mathrm{PhD}^{3}$
}

A man in his 54 was admitted to our hospital owing to progressive postprandial pain for a month. Computed tomography (CT) scan and angiography revealed severe stenosis and calcification of the celiac artery, superior mesenteric artery, and inferior mesenteric artery. Based on the findings of CT scan and angiography, abdominal angina was established and retrograde revascularization was performed only to the superior mesenteric artery using an artificial graft. After the surgery, he remains free of postprandial abdominal pain.

Keywords: abdominal angina, retrograde revascularization

\section{Introduction}

Abdominal angina is a rare disease caused by inadequate blood flow through mesenteric vessels due to stenosis and/or occlusion of one or more mesenteric arteries. ${ }^{1)}$ Because conservative therapy is not effective for abdominal angina, endovascular or surgical procedures are often required in most of the cases. Moreover, the surgical strategies including inflow sites, graft materials, and

${ }^{1}$ Department of Cardiovascular Surgery, Kin-ikyo Central Hospital, Sapporo, Japan

${ }^{2}$ Department of Surgery, Kin-ikyo Central Hospital, Sapporo, Japan

${ }^{3}$ Department of Cardiovascular and Thoracic Surgery, Hokkaido University Hospital, Sapporo, Japan

Received: July 23, 2017; Accepted: October 6, 2017 Corresponding author: Ai Tochikubo, MD. Department of Cardiovascular Surgery, Kin-ikyo Central Hospital, 5-1-9-1 Higashinaebo, Higashi-ku, Sapporo, Hokkaido 007-8505, Japan

Tel: +81-11-782-9111, Fax: +81-11-781-0680

E-mail: ai.tochi716@gmail.com

(cc) BY-NC-SA (02018 The Editorial Committee of Annals of Vascular Diseases. This article is distributed under the terms of the Creative Commons Attribution License, which permits use, distribution, and reproduction in any medium, provided the credit of the original work, a link to the license, and indication of any change are properly given, and the original work is not used for commercial purposes. Remixed or transformed contributions must be distributed under the same license as the original. the number of anastomoses are not fully established for abdominal angina. We successfully treated a patient with abdominal angina by retrograde revascularization with synthetic bypass graft.

\section{Case Report}

A man in his 54 was admitted to our hospital with the complaints of postprandial pain for the last 4 weeks. He had an end-stage renal failure (hemodialysis for 19 years), hypertension, dyslipidemia, and moderate aortic valve stenosis. He had a history of abdominal surgery for perforation of the colon diverticulum. Contrast-enhanced computed tomography (CT) revealed severely calcified orifices of the celiac artery (CA), the superior mesenteric artery (SMA), and the inferior mesenteric artery (IMA) (Fig. 1). Angiography demonstrated severe stenotic orifices of the CA and SMA (Fig. 2). A diagnosis of abdominal angina was established due to the stenosis of all the three major abdominal arteries. Further, it was suggested that endovascular procedures might not be suitable due to the severe calcification of all the mesenteric arterial orifices. Therefore surgical revascularization was eventually planned. Pre-operative transthoracic echocardiography revealed moderate aortic valve stenosis (valve area $=1.5 \mathrm{~cm}^{2}$ ). Coronary angiography detected $75 \%$ luminal stenosis in the proximal region of the right coronary artery, which was previously treated with percutaneous coronary intervention. Considering the associated comorbidities, retrograde surgical bypass, which is less invasive than anterograde revascularization, was considered feasible. For the surgery, a ringed heparincoated $5 \mathrm{~mm}$ artificial graft (PROPATEN, Gore-Tex, Flagstaff, Arizona, USA) to minimize the risk of graft kinking was subsequently selected. Direct contact was avoided between the graft and the abdominal organs, by placing the graft in the retro-peritoneum cavity and was anastomosed to the right external iliac artery in an end-to-side approach because all common iliac arteries were previously treated with endovascular treatment. Another side of the graft was similarly anastomosed to the SMA. Following the 
(A)

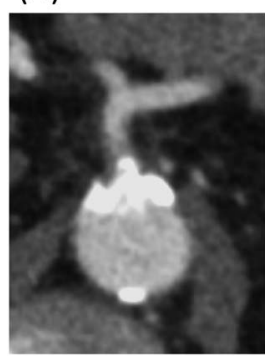

(B)

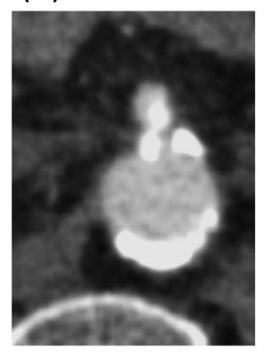

(C)

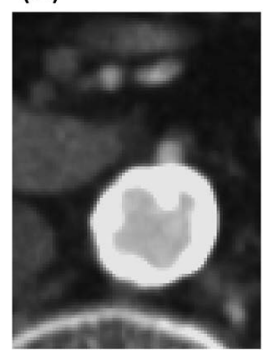

Fig. 1 Pre-operative findings of contrast-enhanced computed tomography. Each orifice is severe calcified: (A) celiac artery; (B) superior mesenteric artery; (C) inferior mesenteric artery.

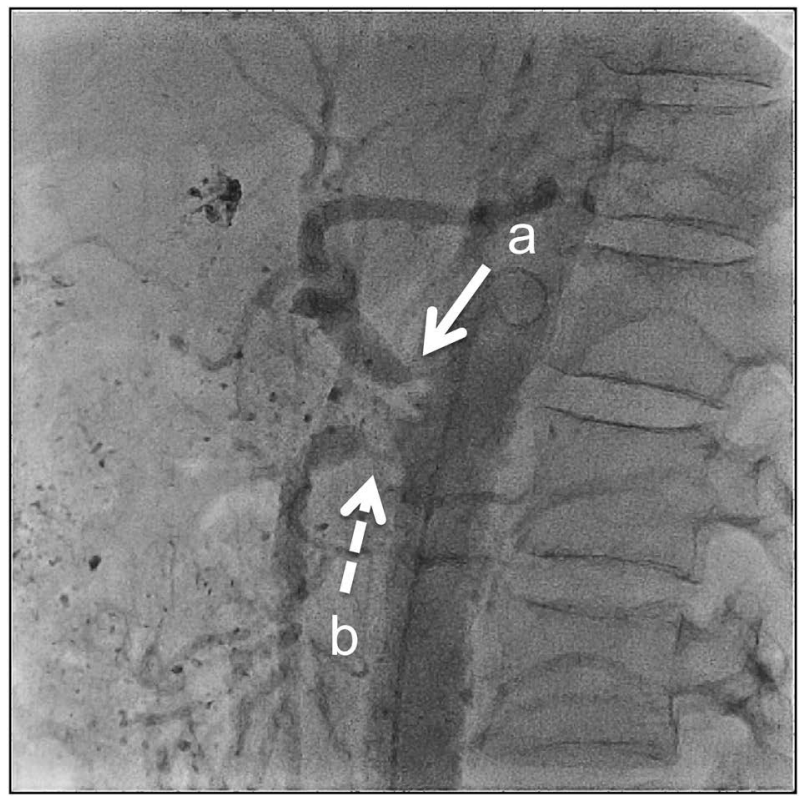

Fig. 2 Pre-operative angiographic findings. The white arrows indicate the stenotic orifices of the celiac artery (a) and superior mesenteric artery (b).

surgery, there was a significant increase in the SMA blood flow volume from 35 to $88 \mathrm{ml} / \mathrm{min}$. Adequate SMA blood flow presumed $40 \mathrm{ml} / \mathrm{min}$ approximately for the patient; thus, we believed that this procedure was satisfactory. ${ }^{1)}$

Oral aspirin and cilostazol were initiated on the next day after the surgery. He remains free of postprandial pain after the procedure. Further, we confirmed the graft patency by CT scan before the discharge (Fig. 3). He was discharged home 14 days after the surgery. We have also established the graft patency rates of 2 years following the surgery.

\section{Discussion}

\section{Pathology of abdominal angina}

Abdominal angina is a rare condition because the diges-

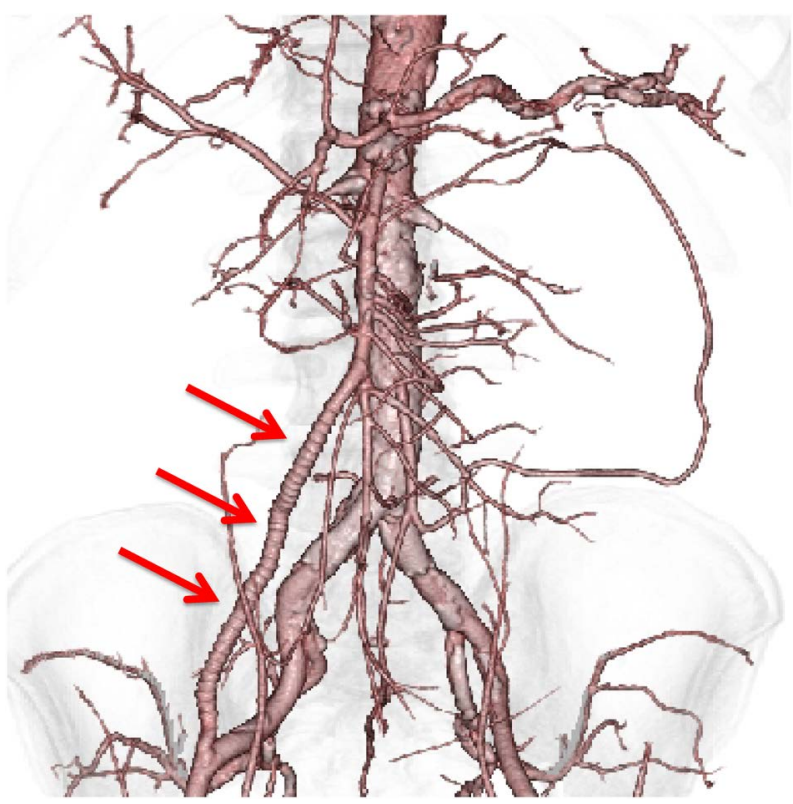

Fig. 3 Post-operative findings of contrast-enhanced computed tomography. The arrows show the patent artificial graft onto the superior mesenteric artery.

tive tract is tolerant for ischemia by blood supply from the CA, SMA, and IMA. ${ }^{2)}$ A significant reduction in the blood flow of at least two of the major mesenteric arteries (CA, SMA, and IMA) can induce abdominal angina. ${ }^{3)}$ Delayed treatment may induce acute mesenteric ischemia; therefore, early and precise diagnosis and prompt treatment are necessary for abdominal angina. The symptoms of abdominal angina include progressive postprandial pain, remarkable weight loss due to food avoidance, diarrhea, and constipation. However, these symptoms are not specific to abdominal angina and even observed in other digestive diseases. Despite a growing clinical consciousness and a rapid development of laboratory and radiologic techniques, the definitive diagnosis of abdominal angina remains challenging, which could delay its effective management. In the present case, CT scanning and angiography confirmed the calcified and stenotic orifices of the CA, SMA, and IMA. The progressive postprandial abdominal pain and hemodialysis indicated the lack of mesenteric blood flow relative to the demand.

\section{Treatment options for abdominal angina}

Therapeutic options for abdominal angina include endovascular procedures and surgical revascularization. Although the endovascular treatment is less invasive than surgery, the rate of restenosis is reported to be high.4) Moreover, surgical procedures are advantageous concerning low recurrence rate of symptoms. ${ }^{5)}$ The treatment strategy should be selected based on the pre-operative patient's comorbidities and conditions. Because of the severe orifices calcification, it was difficult to obtain a 
complete opening of the arteries by endovascular procedures. Therefore, a surgical revascularization was chosen in spite of the history of an abdominal surgery and cardiac comorbidities.

\section{Surgical strategy}

Surgical revascularization includes endarterectomy and bypass surgery. There have been some reports about decent graft patency rate with bypass procedure. ${ }^{2,6)}$ However, the surgical strategies including inflow sites, graft materials, and a number of anastomoses are not fully established. In an anterograde bypass, the inflow graft is anastomosed onto the abdominal aorta above the CA. As it accomplishes less angulated and shorter bypass route than the retrograde one, it is usually unsuitable for highrisk patients as a supra-celiac approach is necessary. However, in retrograde bypass, a longer graft is required and could cause graft kinking. Nonetheless, the graft patency rate at 5 years has been reported to be equal between the anterograde and retrograde bypasses. ${ }^{7}$ In the present case, we selected less invasive retrograde revascularization because he exhibited several comorbidities (untreated moderate aortic valve stenosis and long-time history of hemodialysis). In the retrograde bypass, artificial graft is recommended to avoid graft kinking. ${ }^{8)}$ Additionally, in comparison to autologous vein graft, a synthetic graft has no limitation with suitable length. However, it is highly vulnerable to infection. Furthermore, graft selection remains controversial and mostly depends on baseline characteristics of the patient. We previously experienced autologous vein graft occlusion after retrograde bypass in another patient. Furthermore, he had coronary atherosclerosis. Therefore, we preserved autologous vein graft and decided to use the artificial graft.

Single versus multiple anastomoses is another controversial issue for surgical strategy. In the single bypass strategy, SMA revascularization may have the priority because postprandial mesenteric blood flow changes more abundantly in the SMA. ${ }^{1)}$ Furthermore, isolated bypass onto the SMA is as durable as multi-vessels reconstruction because extensive collateral circulation usually exists among the mesenteric arteries. ${ }^{9)}$ Multiple revascularizations contribute to less recurrent rate after treatment has also been reported. ${ }^{10)}$ In the present case, single revascularization of the SMA was sufficient to improve his symptoms even when all the mesenteric arteries had severe stenosis. Since he had untreated aortic valve stenosis and coronary artery disease, which could both induce mesenteric hypo-perfusion during hemodialysis, the recurrence of symptoms must be carefully followed up.

\section{Conclusion}

We performed a retrograde bypass only to the SMA using an artificial graft for abdominal angina, and the pre-operative symptom disappeared after the surgery. However, a careful follow-up is necessary to evaluate the recurrence of gastrointestinal symptoms.

\section{Disclosure Statement}

All authors have no conflicts of interest to declare.

\section{Author Contributions}

Study conception: SA

Writing: AT

Critical review and revision: all authors

Final approval of the article: all authors

Accountability for all aspects of the work: all authors

\section{References}

1) Nicholls SC, Kohler TR, Martin RL, et al. Use of hemodynamic parameters in the diagnosis of mesenteric insufficiency. J Vasc Surg 1986; 3: 507-10.

2) Rheudasil JM, Stewart MT, Schellack JV, et al. Surgical treatment of chronic mesenteric arterial insufficiency. J Vasc Surg 1988; 8: 495-500.

3) van Lanschot JJ and van Urk H. Vascular reconstruction in intestinal angina. Neth J Surg 1984; 36: 151-5.

4) Oderich GS, Bower TC, Sullivan TM, et al. Open versus endovascular revascularization for chronic mesenteric aschemia: risk-stratified outcomes. J Vasc Surg 2009; 49: 1472-9.e3.

5) Pecoraro F, Rancic Z, Lachat M, et al. Chronic mesenteric ischemia: critical review and guidelines for management. Ann Vasc Surg 2013; 27: 113-22.

6) Kruger AJ, Walker PJ, Foster WJ, et al. Open surgery for atherosclerotic chronic mesenteric ischemia. J Vasc Surg 2007; 46: 941-5.

7) Johnston KW, Lindsay TF, Walker PM, et al. Mesenteric arterial bypass grafts: early and late results and suggested surgical approach for chronic and acute mesenteric ischemia. Surgery $1995 ; 118: 1-7$.

8) Oderich GS, Gloviczki P, Bower TC. Open surgical treatment for chronic mesenteric ischemia in the endovascular era: when it is necessary and what is the preferred technique? Semin Vasc Surg 2010; 23: 36-46.

9) Foley MI, Moneta GL, Abou-Zamzam AM Jr, et al. Revascularization of the superior mesenteric artery alone for treatment of intestinal ischemia. J Vasc Surg 2000; 32: 3747.

10) McAfee MK, Cherry KJ Jr, Naessens JM, et al. Influence of complete revascularization on chronic mesenteric ischemia. Am J Surg 1992; 164: 220-4. 